Artigo Original

Original Article

Emilse Aparecida Merlin Servilha ${ }^{1}$ Marina de Almeida Delatti²

Descritores

Saúde do trabalhador Docentes

Ruído ocupacional Efeitos do ruído

Voz

Distúrbios da voz

Keywords

Occupational health

Faculty

Noise, occupational Noise effects

Voice

Voice disorders

Endereço para correspondência: Emilse Aparecida Merlin Servilha Av. John Boyd Dunlop $s / n^{\circ}$, Jardim Ipaussurama, Campinas (SP), Brasil, CEP: 13060-904.

E-mail: emilsemerlinservilha@ puc-campinas.edu.br

Recebido em: 10/8/2011

Aceito em: 4/7/2012

\section{Percepção de ruído no ambiente de trabalho e sintomas auditivos e extra-auditivos autorreferidos por professores universitários}

\author{
Noise perception in the workplace and auditory and extra- \\ auditory symptoms referred by university professors
}

\section{RESUMO}

Objetivo: Investigar a correlação entre ruído no ambiente de trabalho e sintomas auditivos e extra-auditivos mencionados por professores universitários. Métodos: Oitenta e quatro professores responderam a um questionário com questões sobre identificação, situação funcional e saúde. A relação entre ruído no trabalho e sintomas extra-auditivos e auditivos foi pesquisada. Foi realizada análise estatística, considerando o nível de significância de 5\%. Resultados: Nenhum professor indicou ausência de ruído. As respostas foram agrupadas em Sempre (S) (n=21) e Não Sempre (NS) (n=63). Houve diferença quanto ao pátio e a outra sala de aula como fontes de ruído, classificado como de forte intensidade; acústica insatisfatória e eco. Não houve associação entre referência à presença de ruído e queixas extra-auditivas do tipo digestivo, hormonal, osteoarticular, dentário, circulatório, emocional e respiratório. Não houve associação entre autopercepção de presença de ruído e de queixas auditivas e o grupo $\mathrm{S}$ mostrou maior ocorrência de respostas em incômodo ao ruído, dificuldade de ouvir e tontura/vertigem, zumbido e dor de ouvido. Houve associação entre autopercepção de alterações na voz e ruído e o grupo NS apresentou maior porcentagem de casos com alteração na voz que o grupo S. Conclusão: O ambiente universitário foi considerado ruidoso, porém não houve associação com doenças extra-auditivas e auditivas. As queixas auditivas foram mais evidentes naqueles professores que referiram ruído na modalidade Sempre. A saúde dos docentes é produto multidimensional, desta forma, o ruído não pode ser considerado fator único de agravo.

\begin{abstract}
Purpose: To investigate the correlation between noise in the work environment and auditory and extra-auditory symptoms referred by university professors. Methods: Eighty five professors answered a questionnaire about identification, functional status, and health. The relationship between occupational noise and auditory and extra-auditory symptoms was investigated. Statistical analysis considered the significance level of 5\%. Results: None of the professors indicated absence of noise. Responses were grouped in Always (A) $(n=21)$ and Not Always (NA) ( $n=63)$. Significant sources of noise were both the yard and another class, which were classified as high intensity; poor acoustic and echo. There was no association between referred noise and health complaints, such as digestive, hormonal, osteoarticular, dental, circulatory, respiratory and emotional complaints. There was also no association between referred noise and hearing complaints, and the group A showed higher occurrence of responses regarding noise nuisance, hearing difficulty and dizziness/vertigo, tinnitus, and earache. There was association between referred noise and voice alterations, and the group NA presented higher percentage of cases with voice alterations than the group A. Conclusion: The university environment was considered noisy; however, there was no association with auditory and extra-auditory symptoms. The hearing complaints were more evident among professors in the group A. Professors' health is a multi-dimensional product and, therefore, noise cannot be considered the only aggravation factor.
\end{abstract}

Trabalho realizado na Faculdade de Fonoaudiologia, Pontifícia Universidade Católica de Campinas - PUCCampinas - Campinas (SP), Brasil.

Financiamento: Fundo de Apoio à Iniciação Científica - FAPIC/Reitoria

(1) Faculdade de Fonoaudiologia, Pontifícia Universidade Católica de Campinas - PUC-Campinas - Campinas (SP), Brasil.

(2) Faculdade de Medicina, Pontifícia Universidade Católica de Campinas - PUC-Campinas - Campinas (SP), Brasil.

Conflito de interesses: Não 


\section{INTRODUÇÃO}

A presença do ruído tem sido constantemente abordada no âmbito profissional, especialmente aquele de alta intensidade. Nas escolas, apesar da presença de ruído, suas características são divergentes daquelas encontradas nas empresas, sendo que algumas leis podem, de maneira indireta, colaborar com o professor ${ }^{(1,2)}$ uma vez que estabelecem os níveis de conforto acústico em locais de ensino e naqueles em que se desenvolvem trabalhos que exijam concentração.

As investigações sobre ruído nas escolas têm utilizado como recurso metodológico a avaliação objetiva ${ }^{(3,4)}$ ou subjetiva $^{(5,6)}$ deste fator de risco, ou ainda, a combinação de ambas, de modo a elucidar sua intensidade e frequência, assim como suas consequências sobre a saúde dos participantes.Embora a mensuração objetiva dos níveis de ruído resulte em dados que permitem a comparação dos mesmos com os níveis aceitáveis para cada ambiente de trabalho, não se pode esquecer que é o trabalhador que estará sob este fator de risco e sua percepção e reação a ele fornecem informações valiosas.

As investigações sobre ruído em escolas têm sido mais frequentes nos níveis iniciais e médios de escolarização ${ }^{(5-8)} \mathrm{e}$ menos nas universidades ${ }^{(4,9)}$. Este fato pode se justificar, pois espera-se que no ensino superior os níveis de ruído sejam bastante reduzidos por se tratar de alunos adultos, pelos horários de intervalos serem comuns e pelo fato das atividades nele realizadas não preverem brincadeiras, como ocorre nos recreios das escolas infantis.

As pesquisas têm mostrado também que, em relação aos fatores de risco presentes no ambiente de trabalho, o ruído tem sido um dos mais citados pelos professores, inclusive como uma variável que interfere no desenvolvimento da aula, na aprendizagem dos alunos e na comunicação entre eles ${ }^{(3,10-12)}$. As fontes desse ruído na escola têm sido apontadas, mais frequentemente, como o pátio, as vozes dos alunos nos corredores e na própria sala de aula, além dos ruídos provenientes dos alunos utilizando as quadras poliesportivas e dos sons das salas de música ${ }^{(3,11,13,14)}$.

O ruído constante e em alta intensidade pode trazer consequências negativas para a saúde, tais como reações generalizadas ao estresse, hipertensão arterial, aumento da tensão muscular, dificuldades do sono, transtornos neurológicos, problemas digestivos, transtornos comportamentais, cansaço, falta de atenção e concentração ${ }^{(15,16)}$, além de problemas auditivos como zumbido, vertigem, diminuição da audição, incômodo ao ruído ${ }^{(5,7,17)}$ e vocais, como rouquidão, dor de garganta e afonia ${ }^{(14,18,19)}$.

Diante do grande número de estudos sobre ruído e saúde de professores dos níveis de ensino fundamental e médio, mostra-se necessária a investigação desse mesmo tema na universidade. Nessa perspectiva, o objetivo dessa pesquisa foi investigar a correlação entre a presença de ruído no ambiente de trabalho e de sintomas auditivos e extra-auditivos autorreferidos por professores universitários.

\section{MÉTODOS}

Essa pesquisa integra um projeto maior intitulado "Saúde e trabalho na universidade: estratégias para promover a voz do professor" que recebeu aprovação do Comitê de Ética em Pesquisa com Seres Humanos da Pontifícia Universidade Católica de Campinas em 12/11/2009, sob no 885/09.

Participaram desse estudo 85 professores universitários da área da saúde. Houve a exclusão de um questionário, pois o docente deixou de responder a várias questões, impossibilitando o uso de seus dados. Desta forma, a amostra final contou com um total de 84 respondentes, com média de idade de 50 anos $(\mathrm{DP}=10,10$; mediana $=51)$, sendo a idade mínima de 32 e a máxima de 74 anos. Sessenta e três $(75 \%)$ eram do gênero feminino e 21 (25\%) do masculino; 60 (71,4\%) eram casados; 47 (56\%) eram doutores. O tempo de atuação na carreira docente foi variado, com maior expressividade por um período de 21 anos ou mais, com 41 (48,8\%) docentes. Quarenta e oito $(57,1 \%)$ docentes tinham a universidade como seu único local de trabalho, enquanto $36(42,9 \%)$ atuavam também em outro lugar; 37 (44\%) docentes estavam na universidade há 21 anos ou mais.

O questionário Condições de Produção Vocal-Professor $(\mathrm{CPV}-\mathrm{P})^{(20)}$ que inclui informações sobre identificação, situação funcional, aspectos gerais de saúde, hábitos e aspectos vocais foi aplicado em professores universitários, em versão eletrônica. Especificamente, estudou-se as respostas sobre a presença de ruído na escola, origem/intensidade do ruído, acústica da sala e presença de eco, buscando relacioná-las com outras questões de saúde (problemas digestivos, hormonais, na coluna, dentários, circulatórios, emocionais,respiratórios, de audição e outros). A pesquisa previa a mensuração dos ruídos das salas de aula e a comparação dessas medidas com a percepção do ruído pelos docentes,contudo, a análise objetiva do ruído não foi realizada.

Foi acrescentada a questão "Você tem ou já teve alguma alteração na sua voz?" como mais um item de saúde, embora originalmente ela estivesse localizada em outro item do questionário, mais especificamente em Aspectos Vocais. Em relação a esta última questão, as respostas dos professores às três alternativas oferecidas - não, sim, tive e sim, tenho - foram organizadas em duas categorias: não, quando houve a negativa do professor quanto à presença de alteração vocal, e sim, para a menção da presença da alteração no passado e no presente. Esta decisão foi tomada para favorecer a comparação dos grupos.

Os professores foram contatados por meio de um e-mail, no qual eram convidados a participar da pesquisa. Nesta mensagem constava o link, que ao ser acessado, lhes permitia, inicialmente, encontrar somente o Termo de Consentimento Livre e Esclarecido. O professor deveria concordar com as condições, para então visualizar e preencher o questionário. Do total de 320 docentes da área da saúde, 84 (26,87\%) aceitaram o convite e preencheram o instrumento de pesquisa. Houve receptividade dos participantes ao formato eletrônico do instrumento e à possibilidade de preenchê-lo em etapas por meio de dispositivo que salvava as respostas. O questionário pôde ser acessado durante o período de três meses.

Optou-se por privilegiar a análise das respostas dos docentes, pois a percepção do trabalhador sobre os fatores de risco de seu ambiente de trabalho não pode ser negligenciada, uma vez que é ele o mais afetado e também pelo fato de poder ser conscientizado e de estabelecer mudanças de comportamentos e 
atitudes que venham a favorecer sua saúde no ambiente laboral.

Após o término da coleta de dados, constatou-se que, nenhum dos 84 sujeitos mencionou ausência de ruído e apenas cinco deles o manifestaram na modalidade raramente, inviabilizando a divisão entre presença e ausência de ruído. Diante disso, e cientes de que todos os professores tinham em comum a autopercepção da presença de ruído, optou-se por verificar se havia diferenças entre eles, no que se refere a queixas auditivas (dificuldade de ouvir, dor de ouvido, incômodo ao ruído, zumbido, tontura/vertigem) e extra-auditivas (azia, problemas hormonais, circulatórios, emocionais, rinite, amigdalite, asma, entre outros), tomando-se como indicador a frequência com a qual mencionaram a presença desse fator de risco. Assim, as respostas dos docentes foram organizadas em dois grupos: Sempre (S), que englobava a somatória das respostas dos docentes que referiram que o ruído ocorria sempre, e Não Sempre (NS), quando o ruído foi assinalado nas modalidades de frequência "raramente" e "às vezes", obtendo-se assim, 21 (25\%) sujeitos na categoria S e $63(75 \%)$ na categoria NS.

Inicialmente, fez-se a caracterização sociodemográfica dos participantes, utilizando-se o teste t de Student para averiguar a média, mediana e desvio-padrão das idades. Em seguida, para a análise de dados, comparou-se a autopercepção da presença de ruído nos grupos Sempre e Não Sempre com idade, sexo, escolaridade e estado civil, e para tanto foram utilizados os testes Qui-quadrado e Exato de Fisher.Para a comparação entre a origem do ruído, as qualidades deste e as características das salas de aula, que podem favorecê-lo ou restringi-lo, foram empregados os testes Exato de Fisher e Qui-quadrado. Em seguida, comparou-se, utilizando os mesmos testes estatísticos, as queixas auditivas e extra-auditivas indicadas pelos docentes, com a presença de ruído mencionada nas categorias Sempre e Não Sempre. Para finalizar, foi empregado o teste Qui-quadrado a fim dese comparar as queixas de alteração vocal nos dois grupos de professores. O nível de significância utilizado para os testes foi de $5 \%$.

\section{RESULTADOS}

Quanto à presença de ruído na universidade, os professores autorreferiram que ele ocorria em diferentes frequências, sendo que21 (25\%) disseram ser sempre, 58 (69\%) referiram ser às vezes, cinco $(6 \%)$ disseram ser raramente e nenhum deles mencionou nunca.

Ao se comparar a menção de ruído nos grupos S e NS com dados sobre idade, gênero, estado civil e escolaridade dos professores, não houve diferença entre os grupos. Porém, com relação ao gênero, em ambos os grupos (S e NS) as mulheres foram as que mais se queixaram da presença de ruído, com 16 $(76,19 \%)$ e $46(73,02 \%)$ sujeitos, respectivamente, se comparado aos homens, com cinco $(23,81 \%)$ e $17(26,98 \%)$ sujeitos. No que concerne ao estado civil, houve concordância entre os grupos $\mathrm{S}$ e NS, sendo que os que mais referiram ruído foram os casados, com $16(76,19 \%)$ e $44(69,84 \%)$ professores, respectivamente. Houve maior quantidade de docentes titulados queixando-se de ruído sempre (grupo S), ou seja, 15 (71,43\%) doutores e quatro $(19,05 \%)$ mestres. Tal fato também foi observado no grupo NS, em que os doutores foram maioria, com 31 $(49,21 \%)$ docentes, seguidos pelos mestres, com $11(17,46 \%)$ sujeitos e pelos pós-doutorandos, com sete $(11,11 \%)$.

Houve semelhança entre os dois grupos de professores em relação às fontes de ruído mais frequentes na universidade, entretanto, apenas o pátio e outras salas os distinguiram. Além disso, o ruído foi considerado forte e desagradável pelos professores. A maioria do grupo $\mathrm{S}$ e somente cerca de um terço do NS identificou presença de eco em sala de aula, o que indica que os docentes do primeiro grupo consideram as condições das salas de aula menos propícias à realização de ensino do que seus colegas (Tabela 1).

Em relação às queixas extra-auditivas, constatou-se que o grupo NS foi o mais queixoso, apesar de ambos (S e NS) estarem expostos ao ruído. A gastrite ocorreu em igual frequência nos grupos NS e S; os distúrbios emocionais e circulatórios

Tabela 1. Relação entre origem e características do ruído e das salas de aula, segundo os professores

\begin{tabular}{|c|c|c|c|c|c|c|c|c|c|}
\hline \multirow{3}{*}{ Variáveis } & \multicolumn{9}{|c|}{ Ruído no trabalho } \\
\hline & \multicolumn{4}{|c|}{ Não sempre } & \multicolumn{4}{|c|}{ Sempre } & \multirow{2}{*}{$\begin{array}{l}\text { Valor } \\
\text { de } p\end{array}$} \\
\hline & Sim & $\%$ & Não & $\%$ & Sim & $\%$ & Não & $\%$ & \\
\hline \multicolumn{10}{|l|}{ Origem } \\
\hline Pátio & 26 & 41,27 & 37 & 58,73 & 16 & 76,19 & 5 & 23,81 & $0,006^{*}$ \\
\hline Própria sala de aula & 20 & 31,75 & 43 & 68,25 & 10 & 47,62 & 11 & 52,38 & 0,189 \\
\hline Outra sala de aula & 26 & 41,27 & 37 & 58,73 & 14 & 66,67 & 7 & 33,33 & $0,044^{*}$ \\
\hline Vozes das pessoas & 29 & 46,03 & 34 & 53,97 & 12 & 57,14 & 9 & 42,86 & 0,378 \\
\hline Aparelho de som/TV & 6 & 9,52 & 57 & 90,48 & 1 & 4,76 & 20 & 95,24 & 0,674 \\
\hline Outra & 11 & 17,46 & 52 & 82,54 & 6 & 28,57 & 15 & 71,43 & 0,348 \\
\hline \multicolumn{10}{|l|}{ Características do ruído } \\
\hline Ruído forte & 50 & 79,37 & 13 & 20,63 & 21 & 100 & 0 & 0 & $0,032^{*}$ \\
\hline Presença de eco & 17 & 28,81 & 42 & 71,19 & 11 & 57,89 & 8 & 42,11 & $0,022^{*}$ \\
\hline
\end{tabular}

*Valores significativos $(p \leq 0,05)$ - Teste Exato de Fisher e teste Qui-quadrado 
Tabela 2. Relação entre menção de queixas extra-auditivas e presença de ruído nos grupos Não Sempre e Sempre

\begin{tabular}{|c|c|c|c|c|c|c|c|c|c|}
\hline \multirow{3}{*}{ Queixas extra-auditivas } & \multicolumn{9}{|c|}{ Ruído no trabalho } \\
\hline & \multicolumn{4}{|c|}{ Não sempre } & \multicolumn{4}{|c|}{ Sempre } & \multirow{2}{*}{$\begin{array}{l}\text { Valor } \\
\text { de } p\end{array}$} \\
\hline & Sim & $\%$ & Não & $\%$ & Sim & $\%$ & Não & $\%$ & \\
\hline Azia & 18 & 28,57 & 45 & 71,43 & 9 & 42,86 & 12 & 57,14 & 0,225 \\
\hline Refluxo & 14 & 22,22 & 49 & 77,78 & 6 & 28,57 & 15 & 71,43 & 0,554 \\
\hline Gastrite & 15 & 23,81 & 48 & 76,19 & 5 & 23,81 & 16 & 76,19 & 1,000 \\
\hline Outro digestivo & 7 & 11,11 & 56 & 88,89 & 2 & 10,00 & 18 & 90,00 & 1,000 \\
\hline Dentário & 14 & 22,22 & 49 & 77,78 & 3 & 14,29 & 18 & 85,71 & 0,542 \\
\hline Circulatório & 11 & 17,74 & 51 & 82,26 & 1 & 5,00 & 19 & 95,00 & 0,277 \\
\hline Emocional & 19 & 31,15 & 42 & 68,85 & 4 & 20,00 & 16 & 80,00 & 0,337 \\
\hline Rinite & 22 & 34,92 & 41 & 65,08 & 3 & 14,29 & 18 & 85,71 & 0,073 \\
\hline Sinusite & 17 & 27,42 & 45 & 72,58 & 4 & 19,05 & 17 & 80,95 & 0,446 \\
\hline Bronquite & 0 & 0,00 & 63 & 100,0 & 1 & 4,76 & 20 & 95,24 & 0,250 \\
\hline Asma & 0 & 0,00 & 63 & 100,0 & 1 & 4,76 & 20 & 95,24 & 0,250 \\
\hline Resfriado & 25 & 39,68 & 38 & 60,32 & 6 & 28,57 & 15 & 71,43 & 0,361 \\
\hline Outros problemas respiratórios & 5 & 8,06 & 57 & 91,94 & 2 & 9,52 & 19 & 90,48 & 1,000 \\
\hline Outros problemas de saúde & 11 & 18,03 & 50 & 81,97 & 4 & 19,05 & 17 & 80,95 & 1,000 \\
\hline
\end{tabular}

Teste Exato de Fisher e teste Qui-quadrado $(p \leq 0,05)$

Tabela 3. Relação entre queixas auditivas e presença de ruído mencionadas pelos docentes dos grupos Não Sempre e Sempre

\begin{tabular}{|c|c|c|c|c|c|c|c|c|c|}
\hline \multirow{3}{*}{ Queixas auditivas } & \multicolumn{9}{|c|}{ Ruído no trabalho } \\
\hline & \multicolumn{4}{|c|}{ Não sempre } & \multicolumn{4}{|c|}{ Sempre } & \multirow{2}{*}{$\begin{array}{l}\text { Valor } \\
\text { de } p\end{array}$} \\
\hline & Sim & $\%$ & Não & $\%$ & Sim & $\%$ & Não & $\%$ & \\
\hline Dor de ouvido & 2 & 3,17 & 61 & 96,83 & 1 & 4,76 & 20 & 95,24 & 1,000 \\
\hline Incomodo ao ruído & 14 & 22,22 & 49 & 77,78 & 7 & 35,00 & 13 & 65,00 & 0,252 \\
\hline
\end{tabular}

Teste Exato de Fisher $(p \leq 0,05)$

foram mais frequentes no grupo NS, e o distúrbio emocional um dos distúrbios mais referidos pelos professores estudados. Não houve diferença entre os grupos em nenhuma variável pesquisada. (Tabela 2).

Na comparação entre ruído e queixas especificamente auditivas (Tabela 3), nenhuma variável distinguiu os grupos; contudo, diferentemente das queixas extra-auditivas, a presença constante de ruído sentida pelos professores do grupo $\mathrm{S}$ resultou em maior frequência de queixas, tais como incômodo ao ruído, dificuldade de ouvir, tontura e vertigem, zumbido e dor de ouvido, do que aquelas referidas pelo grupo NS. Nesse caso específico, poderia se suspeitar que o maior número de queixas auditivas tenha como causas mais prováveis a exposição e susceptibilidade ao ruído dos professores do grupo $\mathrm{S}$.

A associação entre autopercepção da presença de ruído e alteração de voz também foi encontrada no presente estudo (Tabela 4), contudo, advindo de professores que não se queixaram da presença desse fator de risco de forma sistemática.
Tabela 4. Relação entre autopercepção de alteração na voz e presença de ruído nos grupos Não Sempre e Sempre

\begin{tabular}{|c|c|c|c|c|c|}
\hline \multirow{3}{*}{$\begin{array}{l}\text { Alteração } \\
\text { na voz }\end{array}$} & \multicolumn{4}{|c|}{ Ruído no trabalho } & \multirow{3}{*}{$\begin{array}{l}\text { Valor } \\
\text { de } p\end{array}$} \\
\hline & \multicolumn{2}{|c|}{ Não sempre } & \multicolumn{2}{|c|}{ Sempre } & \\
\hline & $\mathrm{n}$ & $\%$ & $\mathrm{n}$ & $\%$ & \\
\hline Não & 24 & 42,11 & 14 & 73,68 & $0,011^{*}$ \\
\hline Sim & 33 & 57,89 & 5 & 26,32 & \\
\hline
\end{tabular}

* Valores significativos $(p \leq 0,05)$ - Teste Exato de Fisher

\section{DISCUSSÃO}

Preliminarmente, deve-se reiterar que, nesse estudo, os resultados decorrem da autopercepção de docentes universitários sobre o ruído, ou seja, trata-se de um dado de caráter subjetivo, que poderia ser complementado por outros objetivos, oriundos da mensuração do ruído realizada por aparelhos específicos, o 
que não foi contemplado nesta pesquisa por questões técnicas.

Os resultados sobre percepção de ruído na universidade pelos professores desse estudo revelaram que nenhum deles indicou sua ausência e apenas cinco citaram sua ocorrência ocasional. Este dado confirma aqueles da literatura que classificam as instituições de ensino como locais barulhentos ${ }^{(5-8)}$, inclusive nas universidades ${ }^{(4,9)}$, dados obtidos tanto por meio da mensuração objetiva do ruído, quanto pela avaliação dos professores.

O questionário, além de apresentar algumas opções de fontes de ruído para os professores assinalarem, oferecia também um espaço para que eles complementassem este item com novas informações que julgassem necessárias. Houve a menção de ventiladores e alunos nos corredores, origens também muito referidas na literatura da área ${ }^{(3,13)}$. A divisão das fontes de ruído mencionadas pelos professores nas categorias intra e extraclasse mostra que esta última ocorreu em maior frequência, indicando a necessidade de revisão dos espaços em que acontecem atividades que geram ruído, como o convívio dos estudantes, assim como a acústica das salas de aula, de modo a criar condições mais favoráveis ao ensino e à aprendizagem.

Esses resultados confirmam aqueles de outras pesquisas que indicam o alto nível de ruído nas instituições de ensino advindo, principalmente, do pátio da escola, local de encontro, recreação, conversas e relaxamento do alunado ${ }^{(7,13)}$. Tal fato mostra a necessidade de que sejam previstos aspectos ergonômicos na construção das escolas, dentre eles, o estudo do espaço de forma que as diferentes atividades como ensino, convívio social, esportes e outras, possam ocorrer concomitantemente, sem que o ruído gerado interfira em cada uma delas. Outra questão relevante é o fato de que os professores assinalaram que o ruído tem origem na própria sala de aula. Isso poderia ser contornado com a utilização de metodologias ativas de ensino-aprendizagem, como por exemplo, com a realização de pesquisa de um conteúdo em sala de aula ou no laboratório, com seminários de alunos com contribuições dos colegas, com simulação de júris ou debate de um tema polêmico com apresentação de argumentos prós e contras pelos diferentes grupos de alunos, o que os envolveria nas atividades propostas, reduzindo o ruído da classe.

Os professores do grupo $\mathrm{S}$ avaliaram a acústica das salas de aula como insatisfatória, diferentemente do grupo NS que apontou o contrário. Essa variável distinguiu os dois grupos e pode ser justificada pelo fato de os professores que se queixaram do ruído constante terem uma maior percepção de que a acústica ruim prejudica o desenvolvimento das aulas. Por outro lado, aqueles que citaram o ruído como assistemático parecem não sentir o impacto desse problema de forma tão intensa. Um estudo $^{(21)}$ avaliou o conhecimento e as atitudes de 70 professores quanto ao impacto que o ruído de fundo e a reverberação nas classes causa no aprendizado e na percepção da fala do docente pelos estudantes, e constatou que tais professores não tinham conhecimento adequado sobre a acústica das salas de aula, nem tão pouco sobre o modo como isto afeta a percepção da fala e o aprendizado.

Outra investigação ${ }^{(4)}$ mensurou a acústica de 25 salas de aula por docentes de três faculdades utilizando a escala - bom, regular, ruim e muito ruim - e os resultados mostraram que a frequência nas categorias muito ruim e ruim variou de 63,60\% a $33,30 \%$. Desta forma, mostra-se recorrente na literatura a queixa de docentes sobre a acústica das salas de aula e como ela interfere na realização da aula, na voz e na saúde dos professores ${ }^{(11,14,22,23)}$.

O eco em sala de aula foi um problema apontado pelos docentes, em especial do grupo $\mathrm{S}$ e sua importância reside no fato do mesmo interferir na inteligibilidade da fala de professores e alunos, podendo gerar incompreensões e mal entendidos, restringindo a comunicação entre eles, assim como o processo ensino-aprendizagem ${ }^{(12,22)}$.

As porcentagens das queixas extra-auditivas mencionadas foram bastante variadas nos dois grupos, porém foram menores do que aquelas encontradas em outras investigações, no que se refere a problemas emocionais como o estresse ${ }^{(10)}$ e outras queixas como cansaço mental, dor de garganta, rouquidão e irritação ${ }^{(3)}$, que mostraram índices também muito mais altos do que os do presente estudo. As porcentagens encontradas no presente estudo foram semelhantes às de uma pesquisa ${ }^{(7)}$ em que as principais queixas extra-auditivas foram distúrbios digestivos (azia, gastrite, má digestão, alterações menstruais), e foram semelhantes também às de outro estudo, em que, dos 258 docentes pesquisados, apenas um pequeno contingente deles possuía doenças musculoesqueléticas e respiratórias, doenças digestivas e transtornos mentais ${ }^{(8)}$.

Os distúrbios extra-auditivos decorrentes do ruído mais relacionados na literatura são as reações generalizadas ao estresse, dificuldades do sono, transtornos neurológicos, problemas vestibulares, problemas digestivos e transtornos comportamentais ${ }^{(15,16)}$. Cabe lembrar que o ruído a que os docentes estão submetidos em seu trabalho mostra-se bastante diferente daquele derivado de outros tipos de atividades profissionais como metalurgia, tecelagem, dentre outras, cuja intensidade e frequência são exacerbadas e com periodicidade constante, $o$ que poderia justificar os resultados encontrados. Além disso, as queixas elencadas pelos docentes podem não ter o ruído como sua causa predominante, o que necessitaria de um aprofundamento dos dados e foge ao escopo desse estudo, porém, abre novas perspectivas de pesquisa nesse campo.

Em relação às queixas auditivas, os professores do grupo $\mathrm{S}$ foram mais queixosos, dados compatíveis com outro estudo que identificou $65 \%$ de queixas auditivas pelos professores, sendo a hipoacusia a mais comum $(31,25 \%)$, acompanhada ou não de zumbido e/ou vertigem. Destes docentes, 93,75\% ainda referiram ruído excessivo nas salas de aula ${ }^{(5)}$.

Os professores que fizeram autorreferência à alteração de voz foram aqueles que menos se queixaram de ruído, e a associação inesperada entre eles indica a necessidade de se considerar a multifatorialidade que incide sobre a produção vocal e suas alterações, sendo o ruído apenas um deles, que é valorizado de forma mais ou menos acentuada pelos sujeitos da pesquisa.

A relação entre altos níveis de ruído no ambiente escolar e alteração de voz tem sido abordada frequentemente na literatura, pois diante de um ambiente ruidoso, o professor precisa elevar seu volume de voz de forma sistemática, gerando sobresforço fonatório e até alterações de laringe e distúrbios da 
voz. Apesar de esta relação ser compreensível, a associação entre elas tem sido encontrada em algumas pesquisas ${ }^{(4,10,14)}$, e negadas por outras ${ }^{(24,25)}$, não se podendo afirmar, portanto, a relação inequívoca entre presença de ruído e alteração de voz no professor.

É oportuno ressaltar que, se por um lado, a divisão dos grupos pode ter se constituído em um viés do estudo, originário do fato de que toda a amostra de professores tinha como ponto em comum a autopercepção da presença de ruído no local de trabalho, por outro lado, diferentemente de outras investigações que comparam grupos de sujeitos com e sem queixas de ruído, o presente estudo mostra-se inovador ao tomar como parâmetro a periodicidade ou a frequência com que os professores identificam este fator de risco, valorizando a percepção do profissional, pois é a partir dela que ele irá organizar suas atitudes e estratégias para realizar seu trabalho e sentir-se prejudicado ou não em sua saúde. Os resultados obtidos derivam das informações oferecidas pelo professor trabalhador e confirmam os achados de outros estudos que mostram com mensuração do ruído ambiental, a presença de ruído nas escolas, inclusive aquelas de ensino superior ${ }^{(4,9)}$.

\section{CONCLUSÃO}

A associação entre presença de ruído no ambiente de trabalho e sintomas auditivos e extra-auditivos autorreferidos em professores universitários não foi obtida em termos estatísticos, entretanto, constatou-se que os sintomas auditivos, mostraram-se mais prevalentes no grupo de docentes que referiram sua presença como constante.

A associação entre ruído e alteração na voz foi constatada neste estudo pelo grupo de docentes que mencionou sua presença de forma assistemática, indicando que a alteração vocal tem origem multifatorial e que o ruído não pode ser considerado o único fator de risco para a saúde.

As diferentes percepções da presença e frequência do ruído pelos professores parecem anunciar a necessidade de se combinar avaliações objetivas e subjetivas desse fator de risco no ambiente de trabalho e sua associação com problemas de saúde e voz.

\section{REFERÊNCIAS}

1. Brasil. Ministério do Trabalho e Emprego. Normas Brasileiras Regulamentadoras, NBR 10152-2000. Níveis de Ruído para Conforto Acústico. Brasília; 2000. [Internet]. [citado 2010 Nov 6]. Disponível em: http://www.scribd.com/doc/4035856/NBR-10152-2000-Nivel-de-Ruidopara-Conforto-Acustico.

2. Brasil. Ministério do Trabalho e Emprego. Normas Brasileiras Regulamentadoras, NBR 10151-2000. Avaliação de ruídos em áreas habitadas visando o conforto da comunidade. Brasília; 2000. [Internet]. [citado 2010 Nov6]. Disponível em: http://www.scribd.com/ doc/14126784/NBR-10151-Procedimento-Avalicao-Ruido-Habit.

3. Ribeiro ME, Oliveira RL, Santos TM, Scharlach RC. A percepção dos professores de uma escola particular de Viçosa sobre o ruído nas salas de aula. Rev Equilib Corp e Saúde. 2010;2(1):27-45.

4. Cutiva LC, Muñoz AI. Salud vocal de docentes universitarios y condiciones acústicas en una universidad pública en Bogotá. Salud Trab (Maracay). 2009;17(2):97-105.
5. Martins RH, Tavares EL, Lima Neto AC, Fioravanti MP. Surdez ocupacional em professores: um diagnóstico provável. Rev Bras Otorrinolaringol. 2007;73(2):239-44.

6. Jaroszewski GC, Zeigelboim BS, Lacerda A. Ruído escolar e sua implicação na atividade de ditado. Rev CEFAC. 2007;9(1):122-32.

7. Libardi A, Gonçalves CG, Vieira TP, Silvério KC, Rossi D, Penteado RZ. $O$ ruído em sala de aula e a percepção dos professores de uma escola de ensino fundamental de Piracicaba. Rev Dist Comunic. 2006;18(2):16778.

8. Vedovato TG, Monteiro MI. Perfil sociodemográfico e condições de saúde e trabalho dos professores de nove escolas estaduais paulistas. Rev Esc Enferm USP. 2008;42(2):290-7.

9. Colito AH, Teles B, Gomes CS. Ruído em ambiente de estudo e sua influência sobre desempenho acadêmico de estudantes de computação da Unicamp. Rev Ciência do Ambiente On-Line. 2008;4(1):1-5.

10. Gonçalves VS, Silva LB, Coutinho AS. Ruído como agente comprometedor da inteligibilidade de fala dos professores. Produção. 2009;19(3):466-76.

11. Hans RF. Avaliação de ruído em escolas. Rio Grande do Sul: UFRSPROMEC, 2001. [Internet]. [citado 2010 Dez 16]. Disponível em: http:// www.liberato.com.br/upload/arquivos/ 0131010715441616.pdf.

12. Klatte M, Lachmann T, Meis M. Effects of noise and reverberation on speech perception and listening comprehension of children and adults in a classroom-like setting. Noise Health. 2010;12(49):270-82.

13. Woolner P, Hall E. Noise in schools: a holistic approach to the Issue. Int J Environ Res Public Health. 2010;7(8):3255-69.

14. Ilomaki I, Leppanen K, Kleemola L, Tyrmi J, Laukkanen AM, Vilkman E. Relationships between self-evaluations of voice and working conditions, background factors, and phoniatric findings in female teachers. Logoped Phoniatr Vocol. 2009;34(1):20-31.

15. Seligman J, Ibañez RN, Costa EA, Nudelmann AA. Perda auditiva induzida pelo ruído. In: Campos CA, Costa HO. Tratado de otorrinolaringologia. Vol. 2, São Paulo: Roca; 2002.p.119-125.

16. Costa EA, Morata TC, Kitamura S. Patologia do ouvido relacionada com o trabalho. In: Mendes R. Patologias do trabalho. 2a ed. Vol. 2, São Paulo: Atheneu, 2007. p.1253-82.

17. Ferreira LP, Giannini SP, Figueira S, Silva EE, Karmann DF, Souza TM. Condições de produção vocal de professores da prefeitura do município de São Paulo. Dist Comunic. 2003;14(2):275-91.

18. Chen SH, Chiang SC, Chung YM, Hsiao LC, Hsiao TY. Risk factors and effects of voice problems for teachers. J Voice. 2010;24(2):183-90.

19. Bovo R, Galceran M, Petruccelli J, Hatzopoulos S. Vocal problems among teachers: evaluation of a preventive voice program. J Voice. 2007;21(6):705-22.

20. Ferreira LP, Giannini SP, Latorre MR, Zenari MS. Distúrbio de voz relacionado ao trabalho: proposta de um instrumento para avaliação de professores. Dist Comunic. 2007;19(1):127-36.

21. Ramma L. Knowledge and attitudes of teachers regarding the impact of classroom acoustics on speech perception and learning. S Afr J Commun Disord. 2009;56:35-47.

22. Dreossi RC, Momensohn-Santos TM. O ruído e sua interferência sobre estudantes em uma sala de aula: revisão de literatura. Pró-Fono. 2005;17(2): 251-8.

23. Gonçalves VSB, Sena L, Carvalho M, Silva LB. Ruído ocupacional e a inteligibilidade em salas de aula. 2006. [Internet]. [citado 2010 Dez 16]. Disponível em: http://www.higieneocupacional.com.br/download/ruidovaleria.pdf.

24. Kooijman PG, Jong FI, Thomas G, Huinck W, Donders R, Graamans K, et al. Risk factors for voice problems in teachers. Folia Phoniatr Logop. 2006;58(3):159-74.

25. Alves LP, Araújo LT, Xavier Neto JA. Prevalência de queixas vocais e estudo de fatores associados em uma amostra de professores de ensino fundamental em Maceió, Alagoas, Brasil. Rev Bras Saúde Ocup. 2010;35(121):168-75. 